\title{
BMJ Open Understanding factors critical to the implementation of ehealth in chronic disease management: a realist review protocol
}

\author{
Nida Shahid (D , ${ }^{1}$ Valeria E Rac, ${ }^{1,2}$ Joanna Bielecki, ${ }^{2}$ Whitney Berta ${ }^{1}$
}

To cite: Shahid N,

Rac VE, Bielecki J, et al. Understanding factors critical to the implementation of ehealth in chronic disease management: a realist review protocol. BMJ Open 2021;11:e048250. doi:10.1136/ bmjopen-2020-048250

- Prepublication history and additional supplemental material for this paper are available online. To view these files, please visit the journal online. To view these files, please visit the journal online (http://dx.doi org/10.1136/bmjopen-2020048250).

Received 20 December 2020 Accepted 23 June 2021

Check for updates

(C) Author(s) (or their employer(s)) 2021. Re-use permitted under CC BY-NC. No commercial re-use. See rights and permissions. Published by BMJ.

${ }^{1}$ Institute of Health Policy, Management and Evaluation, Dalla Lana School of Public Health, University of Toronto, Toronto, Ontario, Canada ${ }^{2}$ Program for Health System and Technology Evaluation, Ted Rogers Centre for Heart Research at Peter Munk Cardiac Centre, Toronto General Hospital Research Institute, University Health Network, Toronto, Ontario, Canada

Correspondence to

Ms Nida Shahid;

nida.shahid@mail.utoronto.ca

\section{ABSTRACT}

Introduction Canadians are living longer, many with multiple chronic conditions. This population of older, frail Canadians continues to grow in size as do concurrent demands for community-based, outpatient and ambulatory models of care. Ideally, a multifaceted, proactive, planned and integrated care model includes ehealth. Although several factors are known to facilitate the implementation of ehealth in chronic disease management (CDM), for example, adequate support, usability, alignment of programme objectives, there is a growing body of inconclusive evidence on what is critical for implementation. We aim to achieve a fulsome understanding of factors critical to implementation by conducting a realist reviewan approach suitable for understanding complex interventions. Our proposed review will identify factors critical to the implementation of ehealth in CDM (heart failure, chronic obstructive pulmonary disease, chronic kidney disease and/or diabetes (type 1 or 2)) without limitations to care setting, language, publication year or geography. Findings will be presented in configurations of contexts, mechanisms and outcomes (CMOs).

Methods and analysis A search strategy will be iteratively developed based on the concepts of 'implementation' and 'adoption' of 'ehealth' interventions used within 'CDM' to identify the peerreviewed and grey literature published before 31 March 2021 from five databases (Medline, Embase, Cochrane, CINAHL and PsychInfo) on ehealth interventions actively involving a healthcare provider for CDM among adults. Data extraction and synthesis will be guided by Realist and Meta-review Evidence Synthesis: Evolving Standards (RAMESES) guidelines informing core concepts of CMOs, and a study output will include a middle-range-theory describing the implementation of ehealth in CDM.

Ethics and dissemination Findings will be published in an open-access peer-reviewed journal and presented at relevant conferences. A multistakeholder (patients, caregivers, healthcare providers and practitioners, decision-makers and policy-makers) perspective will be used in our dissemination approach. No formal ethics approval is required for this review. PROSPERO registration number CRD42020208275.

\section{Strengths and limitations of this study}

- To our knowledge, the proposed realist review on factors critical to the implementation of ehealth in chronic disease management (CDM) is the first of its kind to examine and synthesise evidence without limitations to publication date, geography, language, study design or care setting.

- This will be a comprehensive review building on existing knowledge related to ehealth implementation in heart failure, chronic obstructive pulmonary disease, chronic kidney disease and/or diabetes (type 1 or 2) among adults.

- Our realist approach will facilitate the understanding of all three components of the 'CMO configuration', that is, contexts, mechanisms and outcomes critical to ehealth implementation in CDM.

- Our review will use the Mixed Method Appraisal Tool (2018), a critical appraisal tool tailored for systematic mixed-study reviews permitting appraisal of methodological quality of qualitative, quantitative and mixed-method studies.

- In developing clear and generalisable CMOs, we risk oversimplification of the nuanced and complex nature of implementation processes.

\section{BACKGROUND}

Introduction

The financial and societal burden of chronic disease management (CDM) on Canada's healthcare systems necessitates the successful implementation of innovative care models such as ehealth. The WHO recently reported that, in 2018, chronic conditions were responsible for 41 million $(71 \%)$ deaths worldwide, of which 15 million deaths were premature (30-70 years). ${ }^{1}$ In 2019, 44\% of Canadian adults (aged $>20$ years) reported living with at least one chronic condition, ${ }^{2}$ where the most prevalent include heart disease $(8 \%)$, chronic obstructive pulmonary disease (COPD; $10 \%$ ) and diabetes (11\%). As of 2019, almost 60000 Canadians were diagnosed with heart failure (HF) annually, and an estimated 1 million 
report living with HF are living longer due to improved medication management of their multimorbidities but also experiencing long and frequent hospital stays costing our healthcare system up to $\$ \mathrm{C} 3$ billion annually. ${ }^{3}$ In 2018, approximately 2 million Canadians ( $\geq 35$ years) reported living with COPD, a condition occurring more frequently among those with other chronic conditions (eg, diabetes and hypertension) ${ }^{4}$ costing our system an estimate of $\$$ C1.5 billion per year (2017). ${ }^{5}$ Approximately, $10 \%$ (1511 000) of Canadians reported living with type 1 or type 2 diabetes in 2019-a proportion anticipated to increase to $12 \%$ (1 945000 ) by $2029^{6}$ —and costing our system an estimated $\$ \mathrm{C} 1.5$ billion. In 2013, the estimated total cost of \$C13.1 billion for diabetes, and its complications were projected to grow to $\$ \mathrm{C} 16.9$ billion by 2020 . $^{7}$ In 2017, attributable healthcare costs for new cases of diabetes were predicted to be $\$ \mathrm{C} 15$ billion over a 10 -year period. ${ }^{8}$ Since 2008 , at an increasing rate of $35 \% 4$ million (or 1 in 10) Canadians were reported to be living with kidney disease ${ }^{910}$ with an estimated annual cost of $\$$ C32 billion $^{\mathrm{i}}$ in 2019. ${ }^{11}$

With an average life expectancy of 82 years, Canadians are living longer and many with multiple chronic conditions. ${ }^{12}$ A population of older, frail Canadians that is growing in size coupled with high prevalence rates and associated costs further increases demands for community-based, outpatient and ambulatory models of care that are multifaceted, proactive, planned and integrated care models such as ehealth. ${ }^{13}$ Policy-makers and decision-makers in healthcare administration are strategically supporting the development and adoption of innovative models of care delivery that target improved processes and coordinated care. ${ }^{14} 15$ Broadly defined as 'the use of information and communication technology in healthcare'; ehealth interventions are increasingly relied on to improve self-management of chronic conditions. $^{16}$

\section{Terminology}

Although the term ehealth has come into use since the year 2000, there is no consensus definition of the concept: its definition has varied by function, stakeholders and contexts ${ }^{17}$ and entails overlapping categories related to telemedicine, telehealth and telemonitoring. The concept, along with its definitions, continues to evolve to adjust to the changes in language use and more nuanced conceptualisations, for example, the distinction (or lack of) between telemedicine and telehealth. ${ }^{18}$ Coined in the 1970 s, the term telemedicine describes the provision of healthcare services, clinical information, and education over a distance using telecommunications technology. ${ }^{18} 19$ Early examples include group therapy, nursing interactions, televisits to community health workers, telemetry

${ }^{\mathrm{i}}$ Estimate includes costs attributable to chronic kidney disease and to other medical conditions. and home care. ${ }^{18}$ In more contemporary times, the term telehealth came to be viewed as a more encompassing term definitive of patient-physician teleconsultations, and toward the end of the 1990s, the term grew in popularity and was used synonymously with the older term, telemedicine. ${ }^{18}$ Telemonitoring, or monitoring patients at a distance (sometimes referred to as telehomecare or home telecare), is described as a component of a larger chronic care model entailing disease management and care coordination, allowing patients to assume a greater role in managing their health. ${ }^{20}$ In our proposed review, the term 'ehealth' is used interchangeably with and in reference to telemonitoring, remote monitoring or telemedicine, and refers to interventions used to deliver chronic care involving an active intervention, such as remote monitoring and some type of engagement between the patient and healthcare provider. ${ }^{\text {ii }}$

\section{A complex health intervention}

Ehealth interventions used in CDM involve several dimensions of complexity including interacting components. ${ }^{21}$ These can incorporate a range of treatment modalities (eg, remote monitoring and health coaching), targeting various types of populations and users (eg, clinical and non-clinical healthcare providers, patients and informal caregivers) and generating interactions on multiple levels within a given organisation. ${ }^{21}{ }^{22}$ Such interventions have shown to provide efficient and effective healthcare ${ }^{23}$ while offering an evidence-based approach to providing patient education, timely communication, goal setting and linking dispersed healthcare teams. ${ }^{24}$

The global telemedicine market is expected to grow from approximately \$USD 43.2 billion (2019) to \$USD 104 billion $^{\text {iii }}$ by 2024 and \$USD 123 billion by $2030 .{ }^{25} 26$ North America continues to dominate the overall ehealth industry with $>38 \%$ share. ${ }^{27-29}$ In 2016, an assessment of policies across the UK, Germany, Italy and Spain demonstrated moderate-to-high ratings for the perceived value of ehealth in CDM with the highest rating given for $\mathrm{HF}^{30} \mathrm{In}$ the recent response to the COVID-19 pandemic, there has been a seismic shift towards virtual care by physicians and health systems around the world, ${ }^{31}$ providing non-urgent and chronic care. ${ }^{32}$ For example, a $154 \%$ increase in telehealth visits was reported in the USA during the last week of March 2020 compared with the previous year. ${ }^{33}$ Glazier et $a l^{34}$ studied changes in virtual and office primary care visits in Ontario between March and July 2020 reporting a $28 \%$ decrease (from 7.66 to 5.51 per 1000 people/day) in total primary care visits, a decline by $79.1 \%$ in office visits and a 56-fold increase in virtual care compared with 2019. Bashshur et $a l^{35}$ recently commented on the 'massive conversion' to use of ehealth as a service for not

\footnotetext{
ii We will exclude 'telehealth' (ie, informational websites) or any other forms of delivering health information electronically that does not involve active intervention or engagement between healthcare providers and patients.

${ }^{\text {iii }}$ At a compound annual growth rate of $19.1 \%$.
} 
only patients with chronic disease but also those afflicted with COVID-19 while protecting healthcare professionals, demonstrating its effectiveness in settings other than the clinic and providing remote care without compromising quality and continuity of care.

Despite its promise, implementation of ehealth in CDM over the years has remained uneven and slow and considered 'not yet a major component' of most health systems. ${ }^{36}$ Challenges with implementation often cited include factors such as the rapidly advancing type of technology implemented, the varying contexts within which an intervention is implemented and mechanisms used to support implementation. ${ }^{37}$ In their realist evaluation of contextual factors influencing behaviour change using ehealth to improve cardiovascular disease prevention, Coorey $e t a l^{38}$ write that uniformity and identical conditions of use do not hold for complex health interventions introduced into constantly changing social environments.

\section{Theoretical frameworks}

Our review will draw on the principles of Diffusion of Innovation (DOI) as a classic theory that suggests the adoption of an intervention depends on the social context where diffusion is a process by which a new intervention is communicated to a social system over time. ${ }^{39}$ Implementation is defined as part of a decision-making process of adoption, integration and use of new intervention within a setting. ${ }^{39} 40$ Additionally, we will draw on principles of Dissemination and Implementation Science (DIS) to help identify factors critical to the implementation of ehealth on microlevel, mesolevel and macrolevel of health systems. ${ }^{41}$ We will use DOI and DIS principles to organise and define the concepts of contexts, mechanisms and outcomes (CMOs) related to implementation as described in our screening criteria.

\section{What we know about challenges with ehealth intervention implementation}

Implementation and wide-scale adoption of ehealth in CDM have seen varied success and remain a challenge in the present-day. ${ }^{42}$ In 2005 , the term 'plague of pilots' was introduced in reference to the fact that many ehealth initiatives were being implemented as pilots and not as a routine practice. Ten years later, this issue has been cited again to further emphasise challenges related to the poor methodological quality and limited information available on the topic. ${ }^{43}$ The literature provides some insight on potential barriers and facilitators that can influence implementation; however, the evidence is largely mixed and often tied to low rigour in methodology (eg, small sample sizes). ${ }^{44}$ It is evident that ehealth interventions for this population often rely on complex and interacting roles (eg, families, healthcare professionals, policy-makers and decision-makers) and organisational processes (eg, culture, workflow and governing principles) and that multiple factors at different levels within an organisation can influence implementation. For example, having adequate support, the usability of the technology, and contextual, interpersonal and operational factors, such as geography, motivation or alignment of broader goals and objectives are the factors found to play a role in implementation. ${ }^{45-55}$ Besides, the nature of the intervention itself can pose a challenge to work processes and user perceptions. ${ }^{19}$ For example, a US-based study reported successful implementation when the ehealth intervention was formally structured in improving patient care.$^{19} \mathrm{On}$ the contrary, a large-scale UK-based comparative qualitative study ( $\mathrm{n}=221$ ) found that the uncertainty inherent in the implementation process inhibited the full integration of an ehealth intervention within pre-existing organisational processes. $^{51}$

In addition to conclusive evidence, large-scale implementation requires a deeper understanding of the benefits of using ehealth across different sectors and populations. This can include the cost of technology, the required infrastructure, educating patients in using the intervention, training providers in the collection and interpretation of results and incorporation into routine practice. ${ }^{56}$ The full costs of implementation are frequently underestimated due to budgets focused on the initial deployment or pilot phase of an intervention that may have not considered the resources required for longterm operation. ${ }^{57}$ The rapidly changing landscape of technology requires ehealth interventions to constantly evolve to remain useful, making it continuously challenging yet necessary for scholars to generate a fulsome knowledge-base that can be useful in guiding the development and implementation of ehealth. ${ }^{22}{ }^{42}$ As specific health, policy and structural issues vary across different contexts (eg, geographic region, populations), it is also difficult to generalise research findings from one country to another.

In addition to finding mixed evidence on the implementation of ehealth in CDM, a quick search on Google Scholar for realist reviews on our topic published in the last 5 years further illustrate the need for an updated and comprehensive understanding of ehealth implementation in CDM that will not only take into account all three elements of the CMO configuration (ie, various CMOs) but also synthesise evidence that is applicable to more than one type of chronic condition or care setting. This literature points towards a gap in our understanding of ehealth implementation that involves varying types of users, the modalities of the interventions and settings in which they are implemented (table 1).

More specifically, these findings highlight gaps in the knowledge of various contexts driving the mechanisms underpinning ehealth interventions and the related outcomes. For example, one study identified relationships, fit and visibility as core mechanisms to how ehealth interventions worked ${ }^{58}$; on the contrary, Parker et $a \tilde{l}^{\tilde{9}}$ reported limited insights into the mechanisms underpinning interventions and the impact on patient self-efficacy and selfmanagement. A realist review summarising evidence on ehealth implementation in CDM reported there was no clear relationship between implementation strategies 
Table 1 Realist reviews on the ehealth implementation in CDM

\begin{tabular}{|c|c|c|c|c|c|c|}
\hline $\begin{array}{l}\text { Authors } \\
\text { (year) }\end{array}$ & & Vassilev et $a f^{58}$ & Opoku et al ${ }^{61}$ & Parker et $a l^{59}$ & Varsi et $a l^{60}$ & $\begin{array}{l}\text { Shahid et al } \\
\text { (submitted) }\end{array}$ \\
\hline Study period & & 2009-2014 & Until 2015 & 2009-2018 & 2006-2018 & Until 2021 \\
\hline \multirow{2}{*}{ Study design } & Quantitative & & 0 & $\bullet$ & 0 & 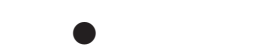 \\
\hline & Mixed-methods & & 0 & 0 & 0 & 0 \\
\hline \multirow{3}{*}{$\begin{array}{l}\text { Disease } \\
\text { population }\end{array}$} & COPD & ○ & & ○ & ○ & ○ \\
\hline & Diabetes & 0 & & ○ & ○ & ○ \\
\hline & CKD & & O & 0 & & 0 \\
\hline $\begin{array}{l}\text { Intervention } \\
\text { description }\end{array}$ & & Telehealth & mHealth & $\begin{array}{l}\text { Ehealth; } \\
\text { mhealth; } \\
\text { telehealth }\end{array}$ & $\begin{array}{l}\text { Clinical } \\
\text { monitoring; } \\
\text { video } \\
\text { consultation; } \\
\text { internet- } \\
\text { delivered } \\
\text { cognitive } \\
\text { therapy }\end{array}$ & $\begin{array}{l}\text { Ehealth, mHealth, } \\
\text { Telemedicine, } \\
\text { Remote } \\
\text { monitoring }\end{array}$ \\
\hline \multirow[t]{3}{*}{ Care-setting } & Primary & & & & & 0 \\
\hline & Community & & & 0 & & 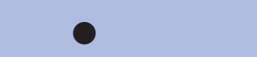 \\
\hline & Home & O & & & ○ & 0 \\
\hline \multirow[t]{3}{*}{$\mathrm{CMO}$} & Contexts & & & & & 0 \\
\hline & Mechanisms & 0 & ○ & 0 & 0 & 0 \\
\hline & Outcomes & & & & $\bullet$ & $\bullet$ \\
\hline
\end{tabular}

CDM, chronic disease management; CKD, chronic kidney disease; $\mathrm{CMO}$, context, mechanism and outcome; COPD, chronic obstructive pulmonary disease; HF, heart failure.

and success. ${ }^{60}$ Similarly, variations in study characteristics reported make it challenging for us to gain a comprehensive understanding of what works, for whom and why when implementing ehealth in CDM? For example, one of the reviews focuses on use of mobile phones in CDM in sub-Sahara Africa without reference to disease type ${ }^{61}$ and another on use of ehealth interventions among disadvantaged patients that include Indigenous/first nation people, culturally and linguistically diverse groups, those experiencing socioeconomic and or geographic disadvantages. ${ }^{59}$ Such heterogeneous evidence further challenges health practitioners and decision-makers in optimising the use of ehealth in CDM and further necessitates a fulsome understanding of implementation processes in real-life settings.

However insightful the body of evidence may be, these findings are mixed and the methodology, population and settings included in extant research are limited in scope. Findings from our review will build on the current evidence without limitations to publication year, language, care setting (eg, primary, community and home), study design and to the extent possible a causal understanding of implementation as evidence-informed theories about the interactions occurring between the contexts and mechanisms of ehealth (as an intervention) and the associated implementation outcomes in configurations of $\mathrm{CMO}{ }^{62}$

\section{Research question}

What CMOs are critical to ehealth implementation in CDM?

\section{Aim}

Building on the existing evidence, our aim is to provide a fulsome synthesis and understanding of the vast and heterogeneous body of literature on the implementation of ehealth in CDM.

\section{Objective}

Our objective will be to assimilate the specific CMOs comprising complex processes of implementation in the form of CMOs that can be resourceful to and applicable by scholars and practitioners alike.

\section{METHODS AND ANALYSIS}

Realist review is a theory-guided approach to understanding complexities of an intervention and generating causal explanations about what works, for whom, in what circumstances and why. ${ }^{63}$ This methodology allows for an 
Table 2 Contexts, mechanisms and outcomes in CDM ehealth implementation

\begin{tabular}{lll}
\hline Concept & Definition & Operationalised examples \\
\hline Context & $\begin{array}{l}\text { Features or conditions relevant to } \\
\text { operation of intervention }\end{array}$ & $\begin{array}{l}\text { Access to and use of patient data in providing appropriate care using } \\
\text { ehealth intervention (use of patient data) }\end{array}$ \\
Mechanism & Often hidden, patterned behaviour & $\begin{array}{l}\text { Use of technology that provides reliable and accurate information and } \\
\text { is interoperable with other clinical systems (intuitive technology) }\end{array}$ \\
Outcome & $\begin{array}{l}\text { Intended/unintended } \\
\text { consequences (can be related to } \\
\text { process or effectiveness) }\end{array}$ & $\begin{array}{l}\text { Improves user acceptance of the ehealth, accessibility and system- } \\
\text { level use of patient data (user acceptance) }\end{array}$ \\
\hline
\end{tabular}

CDM, chronic disease management.

exploration of complexity with a focus on theory generation $^{64}$ often expressed as statements of context, mechanism and outcomes. ${ }^{65}$ Drawing on the principles of DOI and DIS theories, this review will generate evidence-based theory in the form of CMO configurations, on what is it about the implementation of ehealth initiatives used in CDM that works, for whom, in what circumstances and why? ${ }^{64}$

This protocol has been registered in PROSPERO and in compliance with the Preferred Reporting Items for Systematic Review and Meta-Analysis checklist (online supplemental file 1). Study amendments will be tracked and dated in PROSPERO.

\section{Overall design}

Our realist review will adopt an explanatory approach to synthesise and help 'make sense' of the heterogeneous evidence available on the implementation of ehealth in $\mathrm{CDM}^{5166}$ in an attempt to answer our following research question: what CMOs are critical to ehealth implementation in $C D M ?^{67}$ From a realist lens, implementation processes can be influenced by the different contexts in which they occur, resulting in a variety of outcomes. Using a CMO configuration to synthesise the current mixed body of evidence can improve our understanding of the relationships between contexts and causal mechanisms critical to desired implementation outcomes. ${ }^{66} 68$ Pawson and
Tilley ${ }^{68}$ describe contexts as particular circumstances or features of conditions in which interventions are introduced, influencing the operation of mechanisms; mechanisms are often hidden drivers of patterned behaviour that can explain the outcome of an intervention and outcomes as patterns of intended or unintended consequences of different mechanisms occurring in different contexts. Examples of these concepts operationalised for the proposed study are described in table 2.

Adopting Pawson's framework, the stages of our review will be iterative with each stage used to inform the next: (1) clarifying scope, (2) searching and retrieving evidence, (3) quality appraisal and data extraction, followed by (4) analysis and synthesis of the evidence to draw meaningful conclusions $^{6468}$ (figure 1).

\section{Stage 1: clarifying the scope}

To clarify the scope, we (NS and WB) conducted a (unpublished) rapid realist review (RRR) to offer a preliminary understanding of CMOs involved with implementing ehealth in CDM. RRRs are a time-sensitive method used for exploration and knowledge synthesis in consideration of the time and resource limitations of a study. ${ }^{69}$ RRRs have been used to define research questions, streamline the research process and quantify knowledge, that is, understand the amount of literature available identifying CMOs on a given topic, and ensure relevance and

\begin{tabular}{|c|c|c|c|}
\hline 1. Clarify scope & $\begin{array}{l}\text { 2. Search and } \\
\text { retrieve evidence }\end{array}$ & $\begin{array}{l}\text { 3. Data appraisal } \\
\text { and extraction }\end{array}$ & $\begin{array}{c}\text { 4. Data analysis and } \\
\text { synthesis }\end{array}$ \\
\hline $\begin{array}{l}\text { Exploratory } \\
\text { background search } \\
\text { (rapid review) } \\
\\
\text { - Identify review } \\
\text { question, nature of } \\
\text { intervention, and } \\
\text { context for use } \\
\\
\text { - Purpose of review: } \\
\text { Identify } \\
\text { mechanisms, } \\
\text { contexts, and } \\
\text { outcomes critical } \\
\text { to ehealth } \\
\text { implementation }\end{array}$ & $\begin{array}{l}\text { Refine inclusion } \\
\text { criteria based on } \\
\text { emerging data }\end{array}$ & $\begin{array}{l}\text { - Appraise studies } \\
\text { selected for topic } \\
\text { relevance and } \\
\text { rigour } \\
\text { - Use Mixed } \\
\text { Methods Appraisal } \\
\text { Tool (MMAT, } \\
\text { version 2018) } \\
\text { - Develop extraction } \\
\text { form and collect } \\
\text { data (translate if } \\
\text { necessary) }\end{array}$ & $\begin{array}{l}\text { - Descriptive and } \\
\text { thematic analysis } \\
\text { of review findings } \\
\text { - Synthesis data to } \\
\text { develop MRT } \\
\text { - Present } \\
\text { conclusions in } \\
\text { configuration of } \\
\text { CMOs and general } \\
\text { format of 'If A, } \\
\text { then B.' }\end{array}$ \\
\hline
\end{tabular}

Figure 1 Stages of proposed realist review. CMOs, contexts,mechanisms and outcomes; MRT, middle-range-theory. 
applicability of findings based on the evidence base. ${ }^{70} 71$ Our RRR helped narrow the study scope and allowed us to gain a sense of feasibility and whether the body of literature was amenable to our goal of synthesising evidence in CMO configurations. The literature was identified from Medline, Embase and CINAHL between January 2011 and 2016 in addition to a scanning reference lists using the snowball method. After removing articles that were duplicates, non-English or irrelevant, we reviewed 11 articles in full. Based on this initial progress, the overall scope of our review will focus on ehealth interventions actively involving a healthcare provider in self-management of chronic disease among adults.

\section{Stage 2: search and retrieve evidence}

The overall search strategy and syntax will be co-developed and finalised in consultation with a medical information specialist (JB) for the literature published from inception until 31 March 2021. A preliminary search strategy has been conducted to include controlled vocabulary terms and keywords describing the general concepts of 'implementation' and 'adoption' of 'e-health' interventions used within 'chronic disease management'. This search identified the literature from multiple databases related to our query: Ovid Medline (1946-present), Ovid Embase (1947-present), Ebsco CINAHL (1981-present), Wiley Cochrane Library (1996-present) and Ovid PsychInfo (1806-present). The search syntax will be designed and tailored (JB) for each database, results of which will be optimised by iterative examination of the search output for topic relevance (JB and NS; online supplemental file 2).

Following this stage, articles will be screened by one reviewer (NS) and blind-checked by two others (WB and VER) for relevance based on available titles and abstracts and categorised as 'include', 'exclude' or 'maybe' (table 3). Our search will be inclusive of year and language of publication, care setting (eg, primary, community or home) and study design (qualitative, quantitative, mixedmethod or non-empirical). Throughout this stage, we will continue to discuss our screening process and review articles labelled as 'include' or 'maybe' as a team to ensure inner-rater reliability ${ }^{72}$ and resolve disagreements by consensus-based discussions. Owing to the iterative nature of this review, we may revise our search strategy based on the screening results.

The full-text review stage will entail a similar process, where a subset of articles will be independently reviewed by the study team for inclusion or exclusion and discussed as a team for consensus. We will use a review software such as Covidence (www.covidence.org) or citation manager such EndNote (https://endnote.com/) to import citations, screen data for improved efficiency and quality of review.

\section{Stage 3: document appraisal and data extraction}

As part of the appraisal process, each article will be reviewed for relevance (ie, contains information informing our understanding of CMOs) as well as rigour for credible and trustworthy evidence. Based on the nature of empirical

\begin{tabular}{|c|c|c|}
\hline Criteria & Inclusion & Exclusion \\
\hline \multirow{3}{*}{$\begin{array}{l}\text { Publication } \\
\text { type }\end{array}$} & Peer-reviewed and grey literature & Non-peer reviewed literature \\
\hline & $\begin{array}{l}\text { Empirical (qualitative, quantitative and mixed-design) or non- } \\
\text { empirical literature (eg, conceptual or theoretical) }\end{array}$ & $\begin{array}{l}\text { Does not relate to/discuss implementation and/ } \\
\text { or adoption processes and outcomes }\end{array}$ \\
\hline & $\begin{array}{l}\text { Topic relates to implementation processes and outcomes (eg, } \\
\text { acceptability, adoption, appropriateness, cost or feasibility of } \\
\text { an ehealth intervention) }\end{array}$ & $\begin{array}{l}\text { Does not relate to/discuss factors related to the } \\
\text { implementation of ehealth }\end{array}$ \\
\hline Population & $\begin{array}{l}\text { HF, COPD, CKD or diabetes (type } 1 \text { or } 2 \text { ) patients aged } \\
\geq 18 \text { years }\end{array}$ & $\begin{array}{l}\text { Chronic conditions outside study scope } \\
\text { (eg, cancer, mental health) and provided to } \\
\text { populations aged }<18 \text { years }\end{array}$ \\
\hline Setting & Any care setting (eg, primary, community or home) & - \\
\hline
\end{tabular}

CKD, chronic kidney disease; COPD, chronic obstructive pulmonary disease; HF, heart failure. 
studies we expect to be included in our review, we will use the 'Mixed Methods Appraisal Tool' (MMAT, V.2018) for a standardised and transparent appraisal of studies using qualitative, quantitative (randomised controlled trials, non-randomised and descriptive) and mixed-study methodologies $^{73}$ (available online at http://mixedmethods appraisaltoolpublic.pbworks.com).

We will extract and record information related to the (a) general characteristics (author, year and country of publication), (b) purpose and methodology (design, population) used in the literature, (c) aspects of the intervention (eg, remote monitoring device and frequency of consultations) and information available on the (d) CMOs related to the implementation processes described within the article. Data will be extracted by one person (NS) and checked by others (WB and VER). Articles in languages other than English will be reviewed in translation using DeepL Translator (2017), a state-of-the-art translation tool using machine learning techniques (available online at https://wwwdeeplcom/translator). Data will be extracted and managed using Excel.

\section{Stage 4: data analysis and synthesis}

We will use descriptive quantitative analysis to describe the characteristics of our included sources. For the main analysis, we will thematically analyse information and organise it into patterns of CMOs. Data will be synthesised with the overarching goal of improving our understanding of factors playing a critical role in implementation processes in the configurations of CMOs and presented in accordance with RAMESES guidelines. We will aim to include a clear description of how inferences are derived and what information was used to develop and support them. Contradictory data will be used to generate insights about the influence of context. We anticipate that our study findings will offer readers a preliminary middle-range theory(ies) (MRT(s)) specifying what works, for whom and under what conditions concerning our study topic. Although not initiating the realist review with an MRT may be considered by some a departure from realist review literature, initiating one without an MRT still aligns with realist logic and is suitable for answering the open-ended nature of our inquiry (what are the CMOs critical to ehealth implementation in chronic disease management?). ${ }^{67}$

\section{DISCUSSION}

This review aims to provide readers with an improved and comprehensive understanding of factors critical to the implementation of ehealth interventions in CDM among adults in any type of care setting (eg, hospital and home). Explaining the success, failure or mixed results of ehealth interventions as complex interventions can help expand the knowledge base in policy-relevant areas. ${ }^{74}$ Owing to the iterative nature of a realist review, we expect to mitigate any challenges that may arise with the overall methodological process by documentation and transparency. The review may be limited due to a lack of relevant evidence of high rigour. We acknowledge limitations associated with including various study designs in our review, where the inclusion of an article can be subject to interpretation. It will be necessary for the authors to make decisions about relevance based on if and how well the original study findings can contribute to the development of CMO configurations. We anticipate challenges to a certain degree with developing CMO configurations, for example, information extracted as a contextual factor can be simultaneously considered a mechanism or outcome, or an outcome in an initial CMO configuration can later be considered a mechanism in a different configuration. ${ }^{67}$ However, by organising a vastly mixed body of evidence into such configurations, we hope our methodology and findings will help improve the overall transparency and rigour currently reported in monitoring and evaluation of the implementation of complex health interventions in the real-world. We also anticipate our findings will be resourceful to all types of stakeholders (eg, health practitioners, administrators, implementation scientists, decision and policy-makers) by providing a meaningful description of the evidence.

\section{Patient and public involvement}

Members of the public and patients were not involved in the development of this protocol.

\section{Ethics and dissemination}

Ethics approval is not required for this review. Study findings will be disseminated and published in accordance to RAMESES guidelines. ${ }^{74}$ This review will also be published as part of a PhD thesis, available through the University of Toronto TSpace Repository.

Contributors NS and WB: conceived the study. NS is a lead reviewer completing this study as part of a doctoral thesis. NS, WB and VER: co-developed methods for selection, appraisal, extraction and synthesis. JB: iteratively developed the search strategy in consensus with the lead author (NS).

Funding The authors have not declared a specific grant for this research from any funding agency in the public, commercial or not-for-profit sectors.

Competing interests None declared.

Patient consent for publication Not required.

Provenance and peer review Not commissioned; externally peer reviewed.

Supplemental material This content has been supplied by the author(s). It has not been vetted by BMJ Publishing Group Limited (BMJ) and may not have been peer-reviewed. Any opinions or recommendations discussed are solely those of the author(s) and are not endorsed by BMJ. BMJ disclaims all liability and responsibility arising from any reliance placed on the content. Where the content includes any translated material, BMJ does not warrant the accuracy and reliability of the translations (including but not limited to local regulations, clinical guidelines, terminology, drug names and drug dosages), and is not responsible for any error and/or omissions arising from translation and adaptation or otherwise.

Open access This is an open access article distributed in accordance with the Creative Commons Attribution Non Commercial (CC BY-NC 4.0) license, which permits others to distribute, remix, adapt, build upon this work non-commercially, and license their derivative works on different terms, provided the original work is 
properly cited, appropriate credit is given, any changes made indicated, and the use is non-commercial. See: http://creativecommons.org/licenses/by-nc/4.0/.

ORCID iD

Nida Shahid http://orcid.org/0000-0002-8627-6483

\section{REFERENCES}

1 World Health Organization. Noncommunicable diseases country profiles 2018. Geneva: World Health Organization, 2018.

2 Government of Canada. Prevalence of chronic diseases among Canadian adults 2019, 2019. Available: https://www.canada.ca/en/ public-health/services/chronic-diseases/prevalence-canadian-adultsinfographic-2019.html

3 Ted Rogers Centre for Heart Research. By the numbers 2019, 2019. Available: https://tedrogersresearch.ca/by-the-numbers/

4 Government of Canada. Asthma and chronic obstructive pulmonary disease (COPD) in Canada, 2018, 2018. Available: https://www. canada.ca/en/public-health/services/publications/diseasesconditions/asthma-chronic-obstructive-pulmonary-disease-canada2018.html

5 Canadian Institute for Health Information. COPD: a focus on high users - Infographic 2017, 2020. Available: https://www.cihi.ca/en/ copd-a-focus-on-high-users-infographic

6 Diabetes Canada. Diabetes in Ontario 2019, 2019. Available: https:// www.diabetes.ca/DiabetesCanadaWebsite/media/About-Diabetes/ Diabetes Charter/2019-Backgrounder-Ontario.pdf

7 Doucet G, Beatty M. The cost of diabetes in Canada: the economic tsunami. Can J Diabetes 2010;34:27-9.

8 Bilandzic A, Rosella L. The cost of diabetes in Canada over 10 years: applying attributable health care costs to a diabetes incidence prediction model. Health Promot Chronic Dis Prev Can 2017;37:49-53.

9 The Kidney Foundation of Canada. Facing the facts, 2019. Available: https://www.kidney.ca/KFOC/media/images/PDFs/7-3_Facing-theFacts-2019-factsheet.pdf

10 Bello AK, Ronksley PE, Tangri N, et al. Prevalence and demographics of CKD in Canadian primary care practices: a cross-sectional Study. Kidney Int Rep 2019;4:561-70.

11 Manns B, Hemmelgarn B, Tonelli M, et al. The cost of care for people with chronic kidney disease. Can J Kidney Health Dis 2019;6:205435811983552-11.

12 Public Health Agency of Canada. Health status of Canadians 2016. A report of the chief public health officer, 2016. Available: https:// healthycanadians.gc.ca/publications/department-ministere/statepublic-health-status-2016-etat-sante-publique-statut/alt/pdf-eng.pdf

13 MaRS. Tranforming health: shifting from reactive to proactive and predictive care: Mars discovery district, 2019. Available: https://www. marsdd.com/news/transforming-health-shifting-from-reactive-toproactive-and-predictive-care/ - f5

14 Deloitte. Global health care outlook: the evolution of smart health care. London: Deloitte, 2018.

15 Nolte E, Knai C, McKee M. Managing chronic conditions. experience in eight countries (2008): European Observatory on health systems and policies, 2008. Available: https://www.euro.who.int/_data/ assets/pdf_file/0008/98414/E92058.pdf

16 Wildevuur $\bar{S}$, Thomese F, Ferguson J, et al. Information and communication technologies to support chronic disease selfmanagement: preconditions for enhancing the partnership in PersonCentered care. J Particip Med 2017;9:e14.

17 Pagliari C, Sloan D, Gregor P, et al. What is eHealth (4): a scoping exercise to map the field. J Med Internet Res 2005;7:e9.

18 Maheu M, Whitten P, E-Health AA. Telehealth and telemedicine: a guide to Startup and success. New York: John Wiley \& Sons, 2002.

19 Whitten P, Holtz B, Laplante C. Telemedicine: what have we learned? Appl Clin Inform 2010;1:132-41.

20 Bashshur RL, Shannon GW, Smith BR, et al. The empirical foundations of telemedicine interventions for chronic disease management. Telemed J E Health 2014;20:769-800.

21 Craig P, Dieppe P, Macintyre S, et al. Developing and evaluating complex interventions: the new medical Research Council guidance. BMJ 2008;335:a1655.

22 Murray E, Hekler EB, Andersson G. Evaluating digital health interventions: key questions and approaches. Am J Prev Med 2016;51:843-51.

23 Agnihothri S, Cui L, Delasay M, et al. The value of mHealth for managing chronic conditions. Health Care Manag Sci 2020;23:185-202.
24 Davis SM, Jones A, Jaynes ME, et al. Designing a multifaceted telehealth intervention for a rural population using a model for developing complex interventions in nursing. BMC Nurs 2020;19:1-9.

25 BCC Research. Global markets for telemedicine technologies. HLC014K. Wellesley, Massachusetts, USA: BCC Publishing, 2020.

26 BCC Research. Global telemedicine market: analysis and forecast, 2019-2030. BIS385A. Wellesley, Massachusetts, USA: BCC publishing, 2020.

27 ReportLinker. Digital Health Market Research Report: By Technology, End User - Global Industry Analysis and Growth Forecast to 2030, 2020. Available: https://www.reportlinker.com/ p05982506/Digital-Health-Market-Research-Report-By-TechnologyEnd-User-Global-Industry-Analysis-and-Growth-Forecast-to.html? utm_source $=$ GNW

28 Mikulic M. Global telemedicine market size in 2019 and a forecast for 2026 Statista, 2020. Available: https://www.statista.com/statistics/ 671374/global-telemedicine-market-size/

29 Grand View Research. Grand view research. healthcare predictive analytics market analysis by application (operations management, financial, population health, clinical), by end-use (payers, providers), by region (North America, Europe, Asia Pacific, Latin America, MEA) and segment forecasts, 2018-2025, 2016. Available: https://www. grandviewresearch.com/industry-analysis/healthcare-predictiveanalytics-market

30 Rojahn K, Laplante S, Sloand J, et al. Remote monitoring of chronic diseases: a landscape assessment of policies in four European countries. PLoS One 2016;11:e0155738.

31 Webster P. Virtual health care in the era of COVID-19. The Lancet 2020;395:1180-1.

32 Eccleston C, Blyth FM, Dear BF, et al. Managing patients with chronic pain during the COVID-19 outbreak: considerations for the rapid introduction of remotely supported (eHealth) pain management services. Pain 2020;161:889-93.

33 Koonin LM, Hoots B, Tsang CA, et al. Trends in the use of telehealth during the emergence of the COVID-19 pandemic - United States, January-March 2020. MMWR Morb Mortal Wkly Rep 2020;69:1595-9.

34 Glazier RH, Green ME, Wu FC, et al. Shifts in office and virtual primary care during the early COVID-19 pandemic in Ontario, Canada. CMAJ 2021;193:E200-10.

35 Bashshur R, Doarn CR, Frenk JM, et al. Telemedicine and the COVID-19 pandemic, lessons for the future. Telemedicine and eHealth 2020;26:571-3.

36 Barbaellea F, Melchiorre MG, Quattrini S. How can eHealth improve care for people with multimorbidity in Europe? Copenhagen: World Health Organization, Regional Office for Europe, 2016.

37 Tistad M, Lundell S, Wiklund M, et al. Usefulness and relevance of an eHealth tool in supporting the self-management of chronic obstructive pulmonary disease: explorative qualitative study of a Cocreative process. JMIR Hum Factors 2018;5:e10801.

38 Coorey G, Peiris D, Neubeck L, et al. A realist evaluation approach to explaining the role of context in the impact of a complex eHealth intervention for improving prevention of cardiovascular disease. BMC Health Serv Res 2020;20:764.

39 Dingfelder HE, Mandell DS. Bridging the research-to-practice gap in autism intervention: an application of diffusion of innovation theory. $J$ Autism Dev Disord 2011;41:597-609.

40 Parchman M. Diffusion, dissemination and implementation: what is the difference?: group health research Institute 2015.

41 Shelton RC, Lee M, Brotzman LE, et al. What is dissemination and implementation science?: an introduction and opportunities to advance behavioral medicine and public health globally. Int J Behav Med 2020;27:3-20.

42 Saner $\mathrm{H}$. eHealth: from unfulfilled promises to large-scale application. Eur J Prev Cardiol 2016;23:3-4.

43 Urueña A, Hidalgo A, Arenas Álvaro E.. Identifying capabilities in innovation projects: evidences from eHealth. J Bus Res 2016;69:4843-8.

44 Bashi N, Fatehi F, Mosadeghi-Nik M, et al. Digital health interventions for chronic diseases: a scoping review of evaluation frameworks. BMJ Health Care Inform 2020;27:e100066.

45 Carlisle K, Warren R. A qualitative case study of telehealth for inhome monitoring to support the management of type 2 diabetes. $J$ Telemed Telecare 2013;19:372-5.

46 Barriers to Adoption of Information Technology in Healthcare. Proceedings of ACM CASCON concerence. Toronto: Barriers to Adoption of Information Technology in Healthcare, 2017.

47 Hage E, Roo JP, van Offenbeek MAG, et al. Implementation factors and their effect on e-health service adoption in rural communities: a systematic literature review. BMC Health Serv Res 2013;13:19. 
48 Hunting G, Shahid N, Sahakyan Y, et al. A multi-level qualitative analysis of Telehomecare in Ontario: challenges and opportunities. BMC Health Serv Res 2015;15:544.

49 Khoja S, Durrani H, Nayani P, et al. Scope of policy issues in eHealth: results from a structured literature review. J Med Internet Res 2012;14:e34.

50 Kontos E, Blake KD, Chou W-YS, et al. Predictors of eHealth usage: insights on the digital divide from the health information national trends survey 2012. J Med Internet Res 2014;16:e172.

51 May CR, Finch TL, Cornford J, et al. Integrating telecare for chronic disease management in the community: what needs to be done? BMC Health Serv Res 2011;11:131.

52 Ross J, Stevenson F, Lau R, et al. Factors that influence the implementation of e-health: a systematic review of systematic reviews (an update). Implement Sci 2016;11:146.

53 Rousseau DM, House RJ. Meso organizational behavior: avoiding three fundamental biases. Journal of Organizational Behavior 1994:13.

54 Scott Kruse C, Karem P, Shifflett K, et al. Evaluating barriers to adopting telemedicine worldwide: a systematic review. J Telemed Telecare 2018;24:4-12.

55 Ware P, Ross HJ, Cafazzo JA, et al. Evaluating the implementation of a mobile Phone-Based Telemonitoring program: Iongitudinal study guided by the consolidated framework for implementation research. JMIR Mhealth Uhealth 2018;6:e10768.

56 Vegesna A, Tran M, Angelaccio M, et al. Remote patient monitoring via non-invasive digital technologies: a systematic review. Telemed $J$ E Health 2017;23:3-17.

57 World Health Organization. Digital implementation investment guide (DIIG): integrated digital interventions into health programmes. Geneva: World Health Organization, 2020.

58 Vassilev I, Rowsell A, Pope C, et al. Assessing the implementability of telehealth interventions for self-management support: a realist review. Implement Sci 2015;10:59.

59 Parker S, Prince A, Thomas L, et al. Electronic, mobile and telehealth tools for vulnerable patients with chronic disease: a systematic review and realist synthesis. BMJ Open 2018;8:e019192.

60 Varsi C, Solberg Nes L, Kristjansdottir OB, et al. Implementation strategies to enhance the implementation of eHealth programs for patients with chronic illnesses: realist systematic review. J Med Internet Res 2019;21:e14255.
61 Opoku D, Stephani V, Quentin W. A realist review of mobile phone-based health interventions for non-communicable disease management in sub-Saharan Africa. BMC Med 2017;15:24.

62 Jagosh J. Realist synthesis for public health: building an Ontologically deep understanding of how programs work, for whom, and in which contexts. Annu Rev Public Health 2019;40:361-72.

63 Abrams R, Park S, Wong G, et al. Lost in reviews: looking for the involvement of stakeholders, patients, public and other nonresearcher contributors in realist reviews. Research Synthesis Methods 2021;12:239-47.

64 Power J, Gilmore B, Vallières F, et al. Adapting health interventions for local fit when scaling-up: a realist review protocol. BMJ Open 2019;9:e022084.

65 Gordon AL, Devi R, Williams C, et al. Protocol for a realist review of general practitioners' role in advancing practice in care homes (grape study). BMJ Open 2020;10:e036221.

66 Pawson R, Greenhalgh T, Harvey G, et al. Realist review-a new method of systematic review designed for complex policy interventions. J Health Serv Res Policy 2005;10 Suppl 1: 21-34.

67 Jagosh J, Pluye P, Wong G, et al. Critical reflections on realist review: insights from customizing the methodology to the needs of participatory research assessment. Res Synth Methods 2014;5:131-41.

68 Pawson R, Tilley N. Realist evaluation. Thousand Oaks: SAGE, 2004

69 Brown S, Lhussier M, Dalkin SM, et al. Care planning: what works, for whom, and in what circumstances? a rapid realist review. Qual Health Res 2018;28:2250-66.

70 Saul JE, Willis CD, Bitz J, et al. A time-responsive tool for informing policy making: rapid realist review. Implementation Science 2013;8:103.

71 De Weger E, Van Vooren N, Luijkx KG, et al. Achieving successful community engagement: a rapid realist review. BMC Health Serv Res 2018;18.

72 Velonis AJ, Cheff R, Finn D, et al. Searching for the mechanisms of change: a protocol for a realist review of batterer treatment programmes. BMJ Open 2016;6:e010173.

73 Hong QN, Pluye P, Fabregues S. Mixed methods appraisal tool (MMAT), version 2018: Canadian intellectual property office, industry Canada 2018.

74 Wong G, Greenhalgh T, Westhorp G, et al. RAMESES publication standards: realist syntheses. BMC Med 2013;11:21. 